\title{
SUR UNE NOUVELLE FILAIRE D'OISEAUX : PSEUDAPROCTA GUBERNACULARIA N. G., N. SP.
}

\section{Par NADIEJDA SHIKHOBALOV}

Les exemplaires étudiés : trois femelles et un mâle, nous ont étė procurés par I.-A. Dinnik. A notre grand regret la localisation des parasites chez l'hôle n'a pas pu être précisée. Ces helminthes ont étẻ trouvés chez Garrulus krinickii Kaleniczenko dans les environs de Vladikavakase, au nord du Caucase.

Description. - Nématode à corps très grêle, à cuticule très délicatement et transversalement striée. La largeur du corps est presque

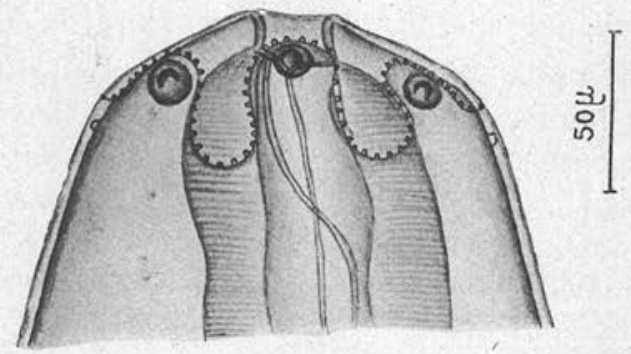

Fıg. 1. - Pseudaprocta gubernacularia n.g. n.sp. - Extrémité céphalique

égale dans toute sa longueur et ne s'atténue que légèrement vers les deux bouts. Les extrémités caudale et céphalique sont arrondies. L'extrémité céphalique est ornée de six grosses papilles : deux de ces papilles sont disposées sur les lignes latérales, deux sur les lignes subventrales et deux sur les lignes subdorsales. Outre ces six grosses papilles céphaliques on distingue encore deux petites papilles, dont l'une est ventrale et l'autre dorsale, disposées à $54 \mu$ de l'extrémité céphalique. Une ornementation fine de petits points scintillants comme de petites perles (fig. 1), forme des festons entre les grandes papilles céphaliques. Cette ornementation est si fine qu'elle est à peine perceptible. La cavité buccale, petite mais bien distincte, est de forme cylindrique. L'œsophage est court, droit et simple.

Annales de Parasitologie, $\mathrm{T}$. ViII, $\mathrm{N}^{\circ} 6 .-1^{\text {er }}$ décembre $1930, \mathrm{p} .624-627$. 
Mâle. - La longueur du mâle est $16 \mathrm{~mm} ., 47$, la largeur maxima $329 \mu$. La longueur de la cavité buccale est de $24 \mu$ et sa largeur de $32 \mu$. L'extrémité caudale (fig. 2), privée d'ailes, est munie à sa pointe d'une petite épine. Les deux spicules sont semblables, de grandeur presque égale. La longueur du plus grand est de $398 \mu$, celle du plus petit de $359 \mu$. Les spicules ont de petites ailes transparentes à peine

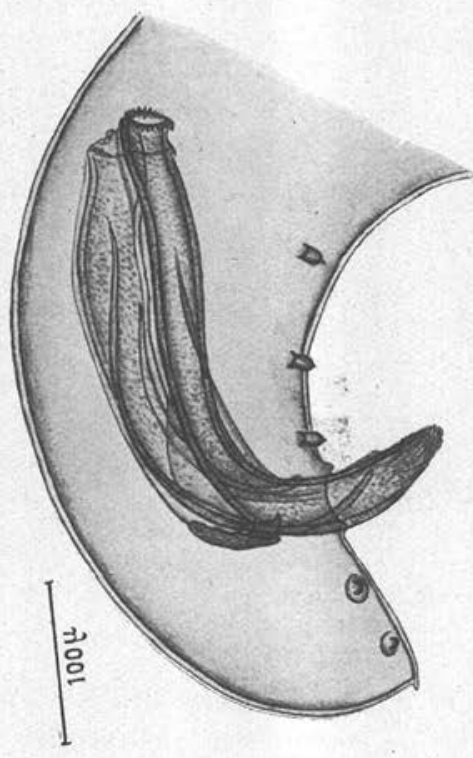

FIG. 2. - Extrémité caudale du mâle.

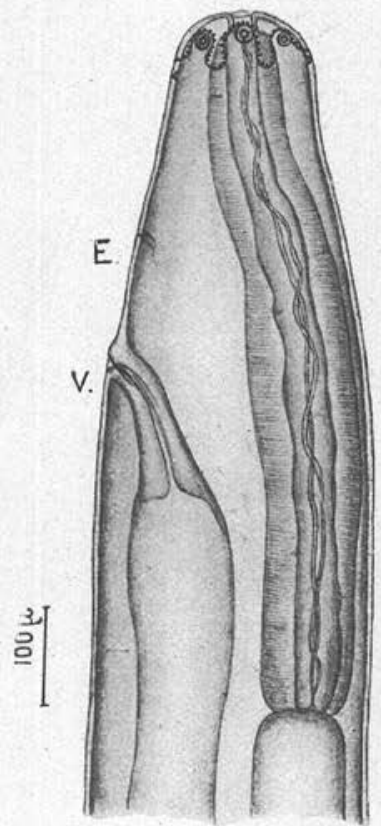

FIg. 3. - Extrémité céphalique de la femelle : $v$, vulve, $e$, pore excréteur.

perceptibles. Le gorgeret est de $72 \mu$ de longueur. On distingue cinq paires de papilles caudales dont trois paires préanales et deux paires postanales.

Femelle. - La longueur du corps est de $16 \mathrm{~mm} ., 47$ à $17 \mathrm{~mm}$., 28, la largeur maxima de 384 à $457 \mu$; au niveau de l'orifice anal, elle est de $290 \mu$. La longueur de la cavité buccale est de $16 \mu$ à $24 \mu$, sa largeur est de $29 \mu$. La longueur de l'œsophage est de 786 à $823 \mu$. La vulve, à fente transversale non saillante, est disposée dans la région de l'œsophage à 402-457 $\mu$ de distance de l'extrémité céphalique (fig. 3). Pore excréteur à $292 \mu$ de l'extrémité antérieure. L'orifice 
anal, bien visible, est éloigné de $52 \mu$ de l'extrémité caudale. Une petite papille arrondie se trouve entre l'extrémité caudale et l'orifice anal, à $28 \mu$ de ce dernier (fig. 4). Sur les exempiaaires étudiés il n’a pas été possible de distinguer ni larves, ni œufs.

Discussion. - La disposition de la vulve dans la région de l'œsophage, les extrémités arrondies du parasite, l'œsophage simple et court, l'orifice buccal sans lèvres, le manque d'ailes sur l'extrémité caudale du mâle, les spicules courts et presque égaux, forment un ensemble de caractères qui nous permettent de placer nos exemplaires dans la famille des Filariidæ et dans la sous-famille des A proc-

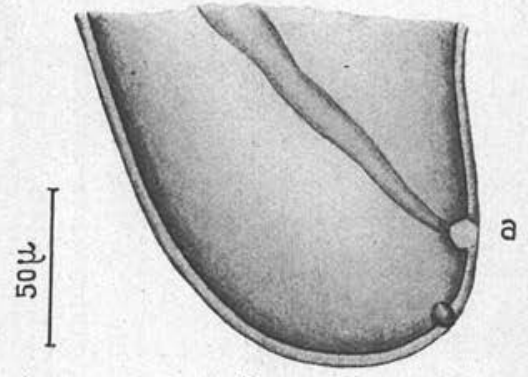

Fıg. 4. - Extrémité caudale de la femelle : $a$, anus

tinæ. Nous ne pouvons pas les placer dans la famille des Setariinæ à cause de l'absence de l'ornementation chitineuse céphalique très exagérée de ces derniers; ils ne peuvent non plus être placés parmi les Diplotriæninæ à cause du manque d'ornementation des dents tricuspides si particulier à ce groupe. La cuticule lisse de nos exemplaires les distingue aussi des Onchocercinæ et Loainæ. La disposition de la vulve dans la région de l'œsophage les sépare des sousfamilles des Crassicaudinæ et Micropleurinæ. Enfin les spicules égaux et subégaux du màle les distinguent de la sous-famille des Filariinæ.

La sous-famille des Aproctinæ embrasse les genres suivants : Pelecitus Raill. et Henry, 1910 ; Coronofilaria Yorke et Maplestone, 1927 ; Chandlerella Yorke et Maplestone, 1927 ; Eufilaria Seurat, 1921 ; Thamugadia, Seurat, 1917 ; Saurositus Macfie, 1924 ; A procta Linstow, 1883.

Le genre Pelecitus est muni d'ailes à l'extrémité caudale des mâles. Les Coronofilaria possèdent un collier buccal ; Thamugadia 
a l'extrémité caudale prolongée et la vulve disposée en arrière de l'œsophage. Tous ces caractères ne nous permettent pas de classer notre espèce parmi l'un de ces genres. Quoique le genre Saurositus possède un gorgeret, néanmoins son œsophage se divise nettement en deux parties distinctes et la vulve de la femelle est disposée juste en arrière de l'osophage ; de plus ce sont des parasites des reptiles. Nous ne pouvons pas classer non plus notre espèce parmi les Eufilaria qui ont un oesophage étroit, transparent, très spécial, ni parmi les Chandlerella dont l'extrémité céphalique est privée de papilles et d'ornementation quelconque et chez lesquels la queue du mâle est allongée et digitiforme.

Les caractères de nos exemplaires les rapprochent le plus des parasites du genre Aprocta. Une bouche simple, un œsophage droit, court et simple, le manque d'ailes chez le mâle, des spicules courts presque égaux, la position de la vulve dans la région de l'osophage, tous ces caractères sont communs avec le genre Aprocta. D'autre part, la présence du gorgeret et du pore anal bien distinct sont en contraste avec ce genre. De toutes les espèces du genre A procta, A procta microanalis Skrjabin, 1917, est seul pourvu d'un rudiment d'anus.

L'extrémité céphalique de nos exemplaires possède une ornementation délicate festonnée et une cavité buccale bien distincte. En outre, la présence d'un pore excréteur, d'un anus et d'un gorgeret ne nous permet pas de les elasser parmi aucun des genres de la sous-famille des Aproctinæx. Cela nous fait ajouter à la liste des genres, un nouveau genre que nous nommerons Pseudaprocta n. g." avec, comme espèce type, et jusqu'à présent unique, $P$. gubernacularia n. sp. du Garrulus krinickii. Voici la diagnose de ce nouveau genre.

Pseudaprocta n. gen. - Aproctinæ. Corps cylindrique, allongé, légèrement atténué vers les deux extrémités. Extrémités arrondies. Cuticule fine, rayée transversalement. Extrémité céphalique pourvue de papilles et d'une ornementation délicate. Pore excréteur présent. Anus bien développé. Extrémité caudale du mâle sans ailes. Papilles caudales présentes. Deux spicules semblables, subégaux. Gorgeret présent. Vulve dans la région de l'œesophage. Parasite d'oiseaux.

Je considère comme mon devoir d'exprimer ma reconnaissance sincère au Prof. K. I. Skrjabine, pour son aide aimable durant mon travail. 